\title{
Heat and Mass Transfer in the Boundary Layer Flow along a Vertical Isothermal Reactive Plate near Stagnation Point: Existence of Dual Solution
}

\author{
G. Singh ${ }^{1 \dagger}$ and P. R. Sharma ${ }^{2}$ \\ ${ }^{1}$ Birla Institute of Technology (Ranchi), Ext. Center Jaipur,27, Malviya Industrial Area, Jaipur-302017, India \\ ${ }^{2}$ Department of Mathematics, University of Rajasthan, Jaipur - 302004, India \\ †Corresponding Author Email: garry_mal@yahoo.com
}

(Received February 2, 2012; accepted March 28, 2013)

\begin{abstract}
Heat and mass transfer in a steady stagnation point boundary layer flow of viscous incompressible fluid through highly porous media along a vertical isothermal plate is investigated. The plate surface reacts with the flowing fluid and inert specie is produced that diffuses inside the boundary layer. The mass flux of the specie at the plate is taken directly proportional to specie concentration at the plate. The governing equations of continuity, momentum, energy and specie diffusion are transformed into ordinary differential equation using the similarity transformation and solved numerically using Runge-Kutta method along with shooting technique. It is found that the system of ordinary differential equations possesses dual solution. The velocity, heat and specie concentration distribution are obtained for different parameters and presented through figures. Skin-friction coefficient, Nusselt number and Sherwood number at plate for various physical parameters are discussed numerically and presented through tables.
\end{abstract}

Keywords: Stagnation point flow, Reactive surface, Specie generation, Dual solution.

\section{NOMENCLATURE}

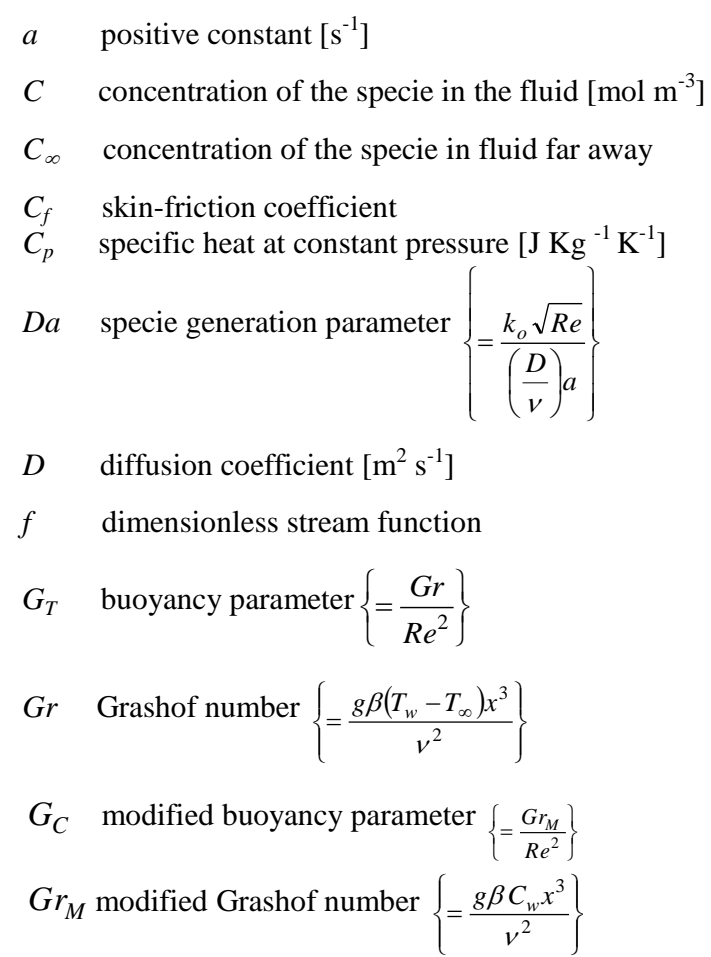

$a \quad$ positive constant $\left[\mathrm{s}^{-1}\right]$

$C \quad$ concentration of the specie in the fluid $\left[\mathrm{mol} \mathrm{m}^{-3}\right]$

$C_{\infty} \quad$ concentration of the specie in fluid far away

$C_{f} \quad$ skin-friction coefficient

$C_{p} \quad$ specific heat at constant pressure $\left[\mathrm{J} \mathrm{Kg}^{-1} \mathrm{~K}^{-1}\right.$

$D a \quad$ specie generation parameter $\left\{=\frac{k_{o} \sqrt{R e}}{\left(\frac{D}{v}\right) a}\right\}$

D diffusion coefficient $\left[\mathrm{m}^{2} \mathrm{~s}^{-1}\right]$

$G_{T}$ buoyancy parameter $\left\{=\frac{G r}{R e^{2}}\right\}$

Gr Grashof number $\left\{=\frac{g \beta\left(T_{w}-T_{\infty}\right) x^{3}}{v^{2}}\right\}$

$G_{C} \quad$ modified buoyancy parameter $\left\{=\frac{G r_{M}}{R e^{2}}\right.$

$G r_{M}$ modified Grashof number $\left\{=\frac{g \beta C_{w} x^{3}}{v^{2}}\right\}$
Re Reynolds number $\left\{=\frac{U_{\infty} x}{v}\right\}$

Sc Schmidt number $\left\{=\frac{v}{D}\right\}$

Sh Sherwood number

$T$ temperature of the fluid [K]

$T_{\infty} \quad$ temperature of the fluid far away from plate [K]

$T_{w} \quad$ temperature of the plate $[\mathrm{K}]$

$u, v \quad$ velocity components along $x$ - and $y$-directions, respectively $\left[\mathrm{ms}^{-1}\right]$

$x, y \quad$ cartesian coordinates $[\mathrm{m}]$ from plate $\left[\mathrm{mol} \mathrm{m}^{-3}\right]$

$\beta^{*} \quad$ coefficient of expansion with concentration $\left[\mathrm{mol}^{-1}\right]$

$\beta \quad$ coefficient of thermal expansion $\left[\mathrm{K}^{-1}\right]$

$\mu \quad$ coefficient of viscosity $\left[\mathrm{kg} \mathrm{m} \mathrm{s}^{-1}\right]$

$\kappa \quad$ coefficient of thermal conductivity $\left[\mathrm{Wm}^{-1} \mathrm{~K}^{-1}\right]$

$\rho \quad$ density of the fluid $\left[\mathrm{kgm}^{-3}\right]$ 
$g \quad$ acceleration due to gravity of the Earth $\left[\mathrm{m} \mathrm{s}^{-2}\right]$

$\tilde{K}$ permeability of porous media $\left[\mathrm{H} \mathrm{m}^{-1}\right]$

$K \quad$ permeability parameter $\left\{=\frac{v}{\tilde{K} c}\right\}$

$k_{0} \quad$ reaction $\operatorname{rate}\left[\mathrm{s}^{-1}\right]$

$\mathrm{Nu}$ Nusselt number

Pr Prandtl number $\left\{=\frac{\mu C_{p}}{\kappa}\right\}$

$R \quad$ interfacial reaction rate constant $\left\{=x k_{0}\right\}\left[\mathrm{ms}^{-1}\right]$ $\phi$ dimensionless concentration $\left\{=\frac{C-C_{\infty}}{C_{\infty}}\right\}$

$\theta$ dimensionless temperature $\left\{=\frac{T-T_{\infty}}{T_{w}-T_{\infty}}\right\}$

$\eta$ dimensionless variable

$v$ kinematic viscosity $\left\{=\frac{\mu}{\rho}\right\}\left[\mathrm{m}^{2} \mathrm{~s}^{-1}\right]$

$\psi$ stream function

\section{INTRODUCTION}

Studies involving the flow along reactive surface has grown due to its implicit occurrence in applications like contamination transport from surfaces, acid mine drainage remediation, waste disposal, fluid rock interaction in hydrothermal system, metal oxide coating of surfaces, reactive flow of gases adjacent to surfaces etc. Example, the fluid is sprayed for cleaning the surface; the contamination at the surface reacts with the fluid and washed away. The processing of micro electro mechanical system (MEMS) requires deposition or selective removal of specie at/from the surface. So, flow along reactive surface also has several utilities in micro/macro engineering, material science, environmental science etc. The above mentioned fluid flow, heat and mass transfer models are parameterized by dimensionless quantities such as Damkohler number, Reynolds number, Prandtl number, buoyancy parameter etc. and hence the interplay among these parameters decides the dynamics of the flow. Chambre and Young (1958) pioneered the study of chemically reacting specie that diffuses inside the boundary layer. Erikson et al. (1966) examined heat and mass transfer with suction or injection on the flat plate. Chin (1975) observed the mass transfer on continuously moving sheet electrode. Murray and Carey (1998) studied the growth of metal oxide on the reactive surface in chemical transport phenomena where the specie concentration is proportional to mass flux at the surface. Succi et al. (2002) analyzed reactive microflows over catalytic surface using Lattice Boltzmann Simulation. Liu et al. (2008) observed the boundary layer modeling of reactive flow over porous surface with angled injection. Chamkha and Aly (2011) studied heat and mass transfer in stagnation point flow of polar fluid through porous media and analysed the effects of Soret $\&$ Dufour and specie consumption. Chamkha and Ahmed (2011) obtained similarity solution to unsteady boundary layer flow of viscous fluid near stagnation point of porous body in the presence of heat generation/absorption and chemical reaction. Hayat et al. (2012) observed mixed convection stagnation point flow of a non-Newtonian fluid on stretching sheet with convective boundary condition. Mahapatra and Nandy (2013) presented mathematical analysis for the magneto hydrodynamic (MHD) axi-symmetric stagnation-point flow and heat transfer over a shrinking sheet with different thermal boundary conditions.
In the present paper, the convective flow of a viscous incompressible fluid in highly porous media near a stagnation point of an isothermal vertical plate is investigated. The plate is reactive to the fluid and produces inert specie, which diffuses inside the boundary layer. The specie concentration at the plate is taken proportional to mass flux at the plate (Murray and Carey, 1998) as the boundary condition. The partial differential equations governing the flow are equations of continuity; momentum, energy and specie diffusion, which are transformed into ordinary differential equations using the similarity transformation and then solved numerically. The interplay between various obtained dimensionless parameters is explained. While converting the reactive surface boundary condition into dimensionless form, a parameter Da, Damkohler number, is obtained which parameterizes the degree of specie generation. $D a$ is the parameter due to which the system of ordinary differential equations has a dual solution.

\section{Formulation Of THE Problem}

Consider a steady laminar stagnation point flow of a viscous incompressible fluid through porous media along a vertical isothermal plate. The plate surface is considered to react with the fluid and produces insert specie that would diffuse into the fluid. The flux of the specie at the plate is taken directly proportional to specie concentration at the plate. The $x$-axis is taken along the plate and $y$-axis is normal to the plate and the flow is confined in half plane $y>0$ as shown through Fig.1.

The potential flow arrives from the $y$-axis and impinges on plate, which divides at stagnation point into two streams and the viscous flow adheres to the plate. The velocity distribution in the potential flow is given by $U_{\infty}(x)=a x$ and $V_{\infty}(y)=-a y$, where $a$ is a positive constant. Following Yih (1998) and Wu et al. (2005), the linear Darcy term representing distributed body force due to porous media is retained while the nonlinear Forchheimer term is neglected, thus the governing equations of continuity, momentum, energy and specie are given by 
$\frac{\partial u}{\partial x}+\frac{\partial v}{\partial y}=0$

$u \frac{\partial u}{\partial x}+v \frac{\partial u}{\partial y}=v \frac{\partial^{2} u}{\partial y^{2}}+g \beta\left(T-T_{\infty}\right)+g \beta *\left(C-C_{\infty}\right)$

$+U_{\infty} \frac{d U_{\infty}}{d x}-\frac{v}{\tilde{K}}\left(u-U_{\infty}\right)$

$u \frac{\partial T}{\partial x}+v \frac{\partial T}{\partial y}=\frac{\kappa}{\rho C_{p}} \frac{\partial^{2} T}{\partial y^{2}}$,

$u \frac{\partial C}{\partial x}+v \frac{\partial C}{\partial y}=D \frac{\partial^{2} C}{\partial y^{2}}$.

The boundary conditions are

$y=0: \quad u=0, v=0, T=T_{w},-D \frac{\partial C}{\partial y}=R C$

[Murray and Carey (1998) ],

$y \rightarrow \infty: u \rightarrow U_{\infty}=a x, T \rightarrow T_{\infty}, C \rightarrow C_{\infty}$.

\section{METHOD OF SOLUTION}

All Introducing the stream function $\psi(x, y)$ such that

$u=\frac{\partial \psi}{\partial y}$ and $v=-\frac{\partial \psi}{\partial x}$,

where $\psi(x, y)=\sqrt{a v} x f(\eta)$ and the dimensionless variable

$\eta=y\left(\frac{a}{v}\right)^{1 / 2}$,

the system of Eq. (1) to Eq. (4) is reduced to system of ordinary differential equations, given as

$$
f^{\prime \prime \prime}+f f^{\prime \prime}-f^{\prime 2}+G_{T} \theta+G_{C} \phi-K\left(f^{\prime}-1\right)+1=0,
$$

$\theta^{\prime \prime}+\operatorname{Pr} f \theta^{\prime}=0$

and

$$
\phi^{\prime \prime}+S c f \phi^{\prime}=0 .
$$

It is observed that the Eq. (1) is identically satisfied.

The boundary condition Eq. (5) are reduced to

$$
\begin{aligned}
& f(0)=0, f^{\prime}(0)=0, f^{\prime}(\infty)=1, \theta(0)=1, \theta(\infty)=0, \\
& \phi^{\prime}(0)=-D a(1+\phi(0)) \text { and } \phi(\infty)=0 .
\end{aligned}
$$

The Eqs. (8), (9) and (10) along with boundary conditions Eq. (11) form a system of coupled non-linear ordinary differential equations. The system is solved using Runge-Kutta fourth order method along with shooting technique (Conte and Boor, (1981); Sharma and Singh, (2010)).

\section{SKIN FRICTION COEFFICIENT}

Skin-friction coefficient at the plate is given by.

$C_{f}=2(R e)^{-1 / 2} f^{\prime \prime}(0)$

\section{NUSSELT NUMBER}

The rate of heat transfer in terms of the Nusselt number at the plate is given by

$N u=-(\operatorname{Re})^{1 / 2} \theta^{\prime}(0)$.

\section{SHERWOOD NUMBER}

The rate of mass transfer in terms of the Sherwood number at the plate is given by:

$S h=-(R e)^{1 / 2} \phi^{\prime}(0)$.

\section{RESUlts AND Discussion}

It is seen from Table 1 that with the increase in parameter $D a$ the skin friction, the rate of heat transfer, the rate of mass transfer and specie concentration at the plate increase. This is explained by a fact that the parameter $D a$ is the specie generation parameter, therefore the increase in $D a$ increases the influx of specie from plate to fluid, which in turn increases the specie concentration in the fluid, as is seen in Fig. 4. The increase in specie concentration in fluid results in the increase in modified buoyancy force and so the fluid velocity increases which is depicted in Fig. 2. The flowing fluid exerts drag (measured as skin friction) on the plate, and as the fluid velocity increases the plate experiences more drag i.e. skin friction increases. Adding, the increase in fluid velocity would mean that heat is convected better and so the fluid temperature decreases with the increase parameter $D a$, as seen in Fig. 3. The decrease in fluid temperature therefore increases the heat influx at the plate.

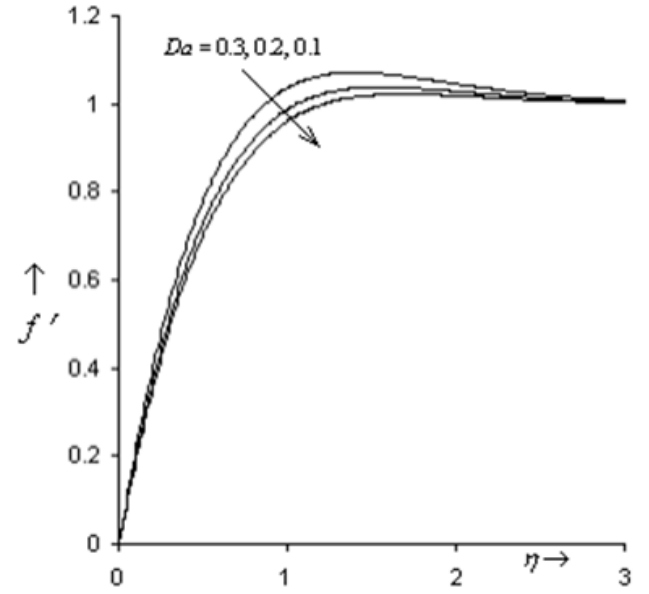

Fig. 2. Velocity distribution versus $\eta$ when $G_{T}=1.0$, $G_{C}=0.5, K=1, \operatorname{Pr}=1.0, S c=0.5$. 


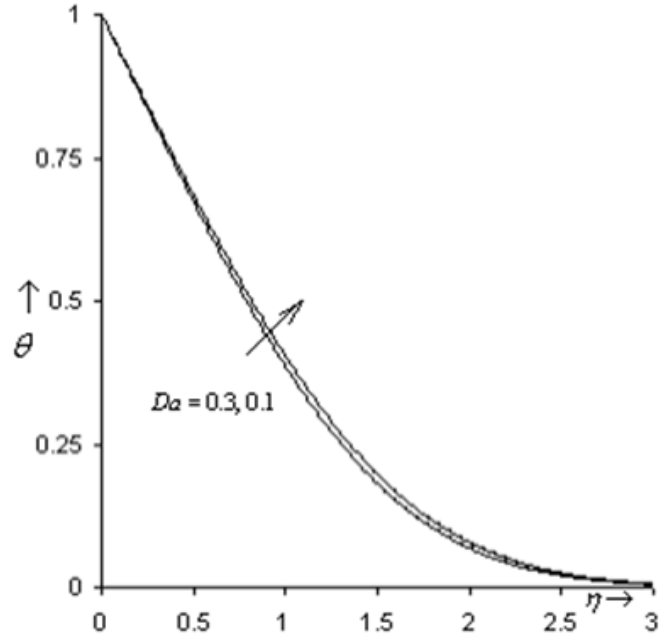

Fig. 3. Temperature distribution versus $\eta$ when $G_{T}=1.0$, $G_{C}=0.5, K=1, \operatorname{Pr}=1.0, S c=0.5$.

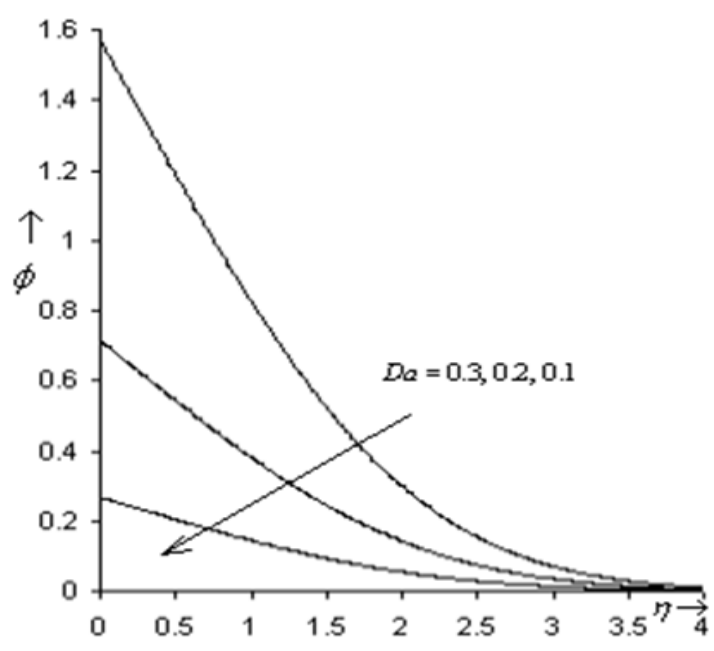

Fig. 4. Concentration distribution versus $\eta$ when $G_{T}=1.0, G_{C}=0.5, K=1, P r=1.0 \& S c=0.5$.

Table 1 Numerical value of $f^{\prime \prime}(0), \theta^{\prime}(0), \phi^{\prime}(0)$ and $\phi(0)$ for different value of parameters.

\begin{tabular}{|c|c|c|c|c|}
\hline & \multicolumn{4}{|c|}{$G_{T}=1.0, G_{C}=0.5, K=1.0, P r=1.0, S c=0.5$} \\
\hline$D a$ & $f^{\prime \prime}(0)$ & $-\theta^{\prime}(0)$ & $-\phi^{\prime}(0)$ & $\phi(0)$ \\
\hline 0.1 & 2.0751 & 0.6346 & 0.1266 & 0.2667 \\
\hline 0.2 & 2.1764 & 0.6430 & 0.3425 & 0.7128 \\
\hline 0.3 & 2.3678 & 0.6583 & 0.7713 & 1.5712 \\
\hline 0.4 & 2.7944 & 0.6902 & 1.8208 & 3.5520 \\
\hline 0.5 & 3.8125 & 0.7568 & 4.8055 & 8.6116 \\
\hline Sc & \multicolumn{4}{|c|}{$G_{T}=1.0, G_{C}=0.5, K=1.0, P r=1.0, D a=1.5$} \\
\hline 10 & 3.0021 & 0.6686 & 13.7553 & 8.1702 \\
\hline 20 & 2.3150 & 0.6398 & 5.7476 & 2.8317 \\
\hline 30 & 2.1911 & 0.6349 & 4.2576 & 1.8384 \\
\hline 40 & 2.1415 & 0.6331 & 3.6410 & 1.4273 \\
\hline 50 & 2.1148 & 0.6321 & 3.2975 & 1.1983 \\
\hline$K$ & \multicolumn{4}{|c|}{$G_{T}=1.0, G_{C}=0.5, P r=1.0, D a=0.2, S c=0.5$} \\
\hline 1 & 2.1764 & 0.6430 & 0.3425 & 0.7128 \\
\hline 5 & 2.9629 & 0.6679 & 0.3356 & 0.6783 \\
\hline 10 & 3.7163 & 0.6852 & 0.3313 & 0.6565 \\
\hline $\operatorname{Pr}$ & \multicolumn{4}{|c|}{$G_{T}=1.0, G_{C}=0.5, K=1.0, D a=0.2, S c=0.5$} \\
\hline 0.71 & 2.1972 & 0.5607 & 0.3412 & 0.7061 \\
\hline 7.0 & 2.0518 & 1.3375 & 0.3487 & 0.7437 \\
\hline 15.0 & 2.0060 & 1.7552 & 0.3502 & 0.7513 \\
\hline$G_{T}$ & \multicolumn{4}{|c|}{$G_{C}=0.5, K=1.0, D a=0.2, P r=1.0 S c=0.5$} \\
\hline 0.5 & 2.1764 & 0.6430 & 0.3425 & 0.7128 \\
\hline 3.0 & 2.9376 & 0.6937 & 0.3274 & 0.6372 \\
\hline 5.0 & 3.6497 & 0.7354 & 0.3172 & 0.5860 \\
\hline$G_{C}$ & \multicolumn{4}{|c|}{$G_{T}=1.0, K=1.0, D a=0.2, \operatorname{Pr}=1.0 S c=0.5$} \\
\hline 0.5 & 2.1764 & 0.6430 & 0.3425 & 0.7128 \\
\hline 2.0 & 2.6004 & 0.6760 & 0.3320 & 0.6601 \\
\hline 4.0 & 3.0756 & 0.7099 & 0.3227 & 0.6135 \\
\hline
\end{tabular}


Table 1 shows that with the increase in Schmidt number $(S c)$ the skin friction, heat transfer, mass flux and the specie concentration at the plate decrease. The Schmidt number is the property of fluid and measures its affinity for specie; larger is the Schmidt number lesser is the affinity to specie. Therefore the specie concentration is higher at lower Schmidt number, which is seen in Fig. 7. Also, the specie concentration at the plate is larger for smaller Schmidt number, which directly implies greater mass flux due to taken initial condition at plate for specie. Further, high specie concentration in fluid increases the modified buoyancy, which increases fluid velocity, as shown in Fig. 5, and so the skin friction is higher at low Schmidt number. Further higher is fluid velocity lesser is the fluid temperature, which can be observed in Fig. 6 and hence higher is the heat flux (heat transfer) at the plate. The parameter $K$ measures the porosity of the medium; higher the value of $K$ lesser is the porosity. As $K$ increases velocity boundary thickness decreases, and thus the velocity and skin friction increases.

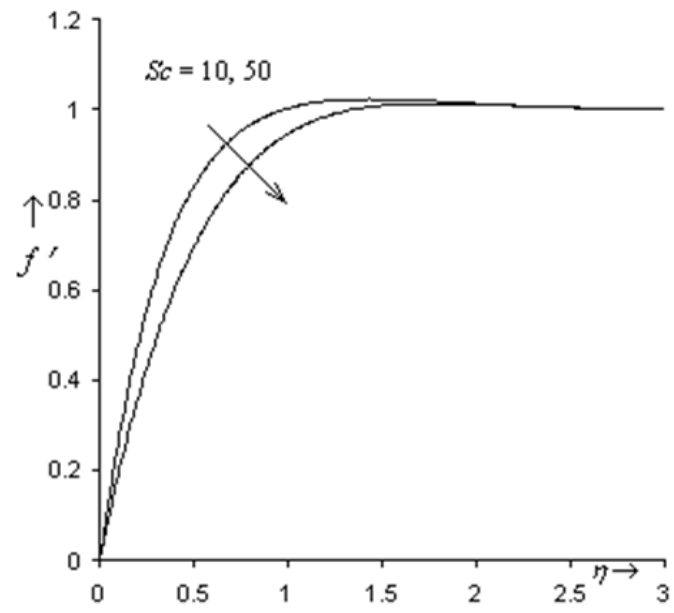

Fig. 5. Velocity distribution versus $\eta$ when $D a=1.5, G_{T}=1.0$, $G_{C}=0.5, K=1 \& P r=1.0$

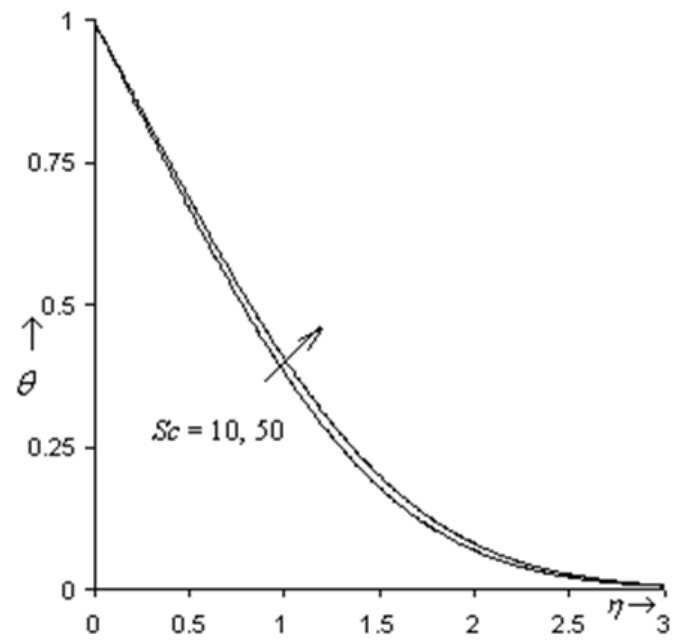

Fig. 6. Temperature distribution versus $\eta$ when $D a=1.5$, $G_{T}=1.0, G_{C}=0.5, K=1 \& \operatorname{Pr}=1.0$

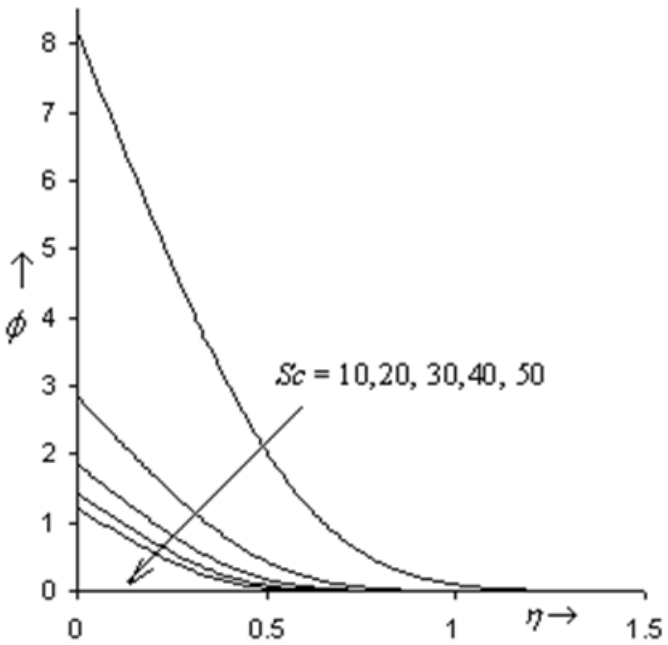

Fig. 7. Concentration distribution versus $\eta$ when $D a=1.5$, $G_{T}=1.0, G_{C}=0.5, K=1 \& \operatorname{Pr}=1.0$

This is observed in Fig. 8 and Table 1, respectively. The increase in fluid velocity leads to better convection, so the fluid temperature decreases at higher value of $K$ and thereby increasing the heat flux.

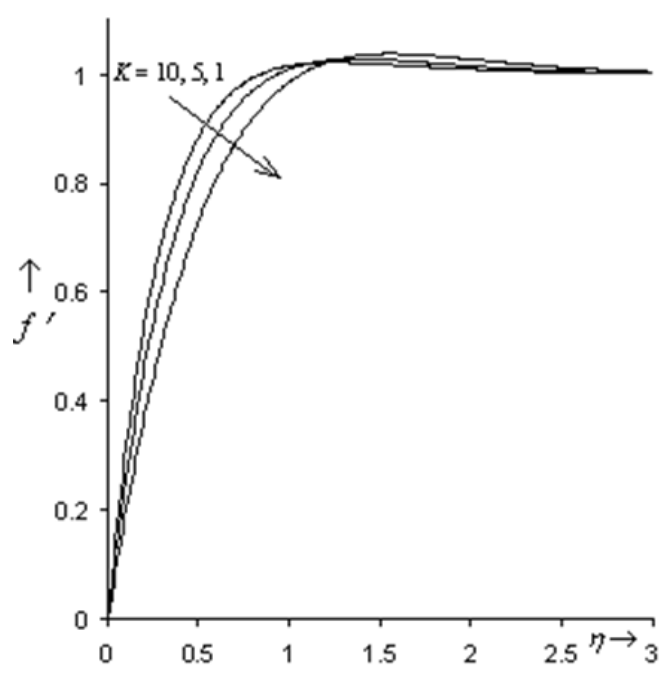

Fig. 8. Velocity distribution versus $\eta$ when $G_{T}=1.0, G_{C}=0.5$, $\operatorname{Pr}=1.0, S c=0.5 \& D a=0.2$.

The same is seen in Fig. 9 and Table 1. Further, Fig. 10 and Table 1 show that specie concentration in the fluid decreases with the increase in fluid velocity due to increase in $K$ and so the mass flux decreases at the plate. Table 1 depicts that with the increase Prandtl number $(P r)$, skin friction decreases, while the rate of heat transfer, mass transfer and specie concentration increase. Prandtl number is the fluid property and measures the thermal conductivity. Smaller is the Prandtl number better is the thermal conductivity and thicker is the thermal boundary layer. So the fluid temperature is high for low Prandtl fluid, as is observed in Fig. 12. The increase in fluid temperature decrease the temperature gradient at the plate thus the rate of heat transfer is less for fluid of low Pr. Higher fluid temperature leads to more buoyancy force (evaluated by $T-T_{\infty}$ ) which increases the fluid velocity and also the 
G. Singh and P. R. Sharma / JAFM, Vol. 7, No. 1, pp. 25-33, 2014.

skin friction in the case of low Prandtl number. This is exactly what is seen in Fig. 11.

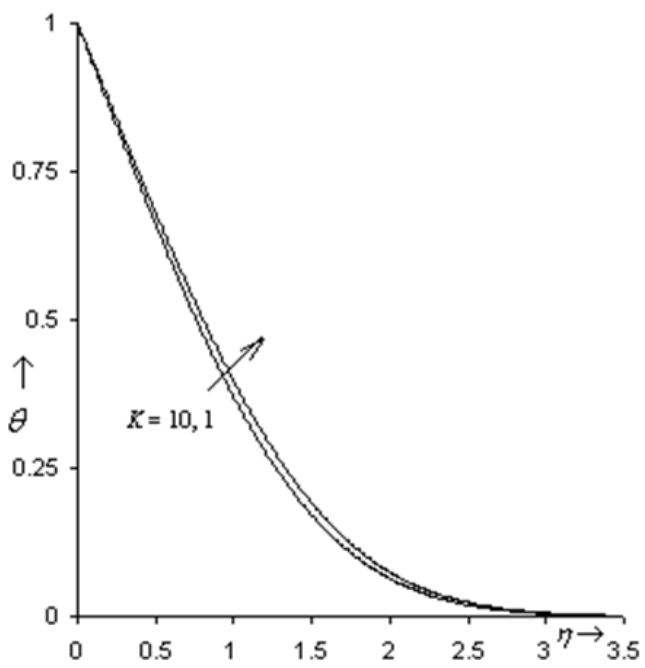

Fig. 9. Temperature distribution versus $\eta$ when $G_{T}=1.0$, $G_{C}=0.5, \operatorname{Pr}=1.0, S c=0.5 \& D a=0.2$.

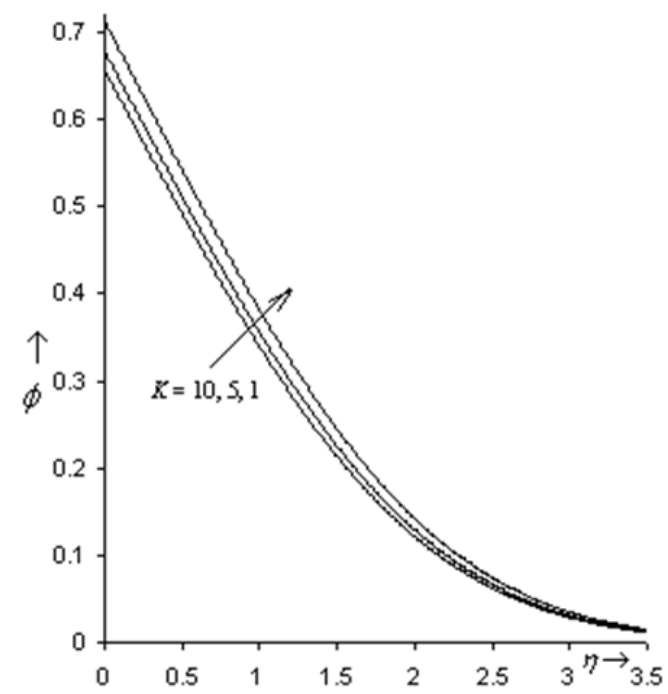

Fig. 10. Concentration distribution versus $\eta$ when $G_{T}=1.0$, $G_{C}=0.5, P r=1.0, S c=0.5 \& D a=0.2$

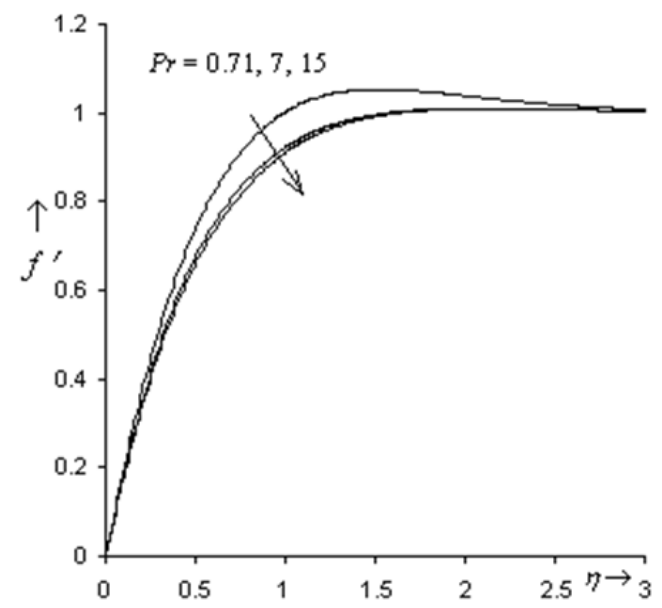

Fig. 11. Velocity distribution versus $\eta$ when $D a=0.2, G_{T}=1.0$, $G_{C}=0.5, K=1 \& S c=0.5$

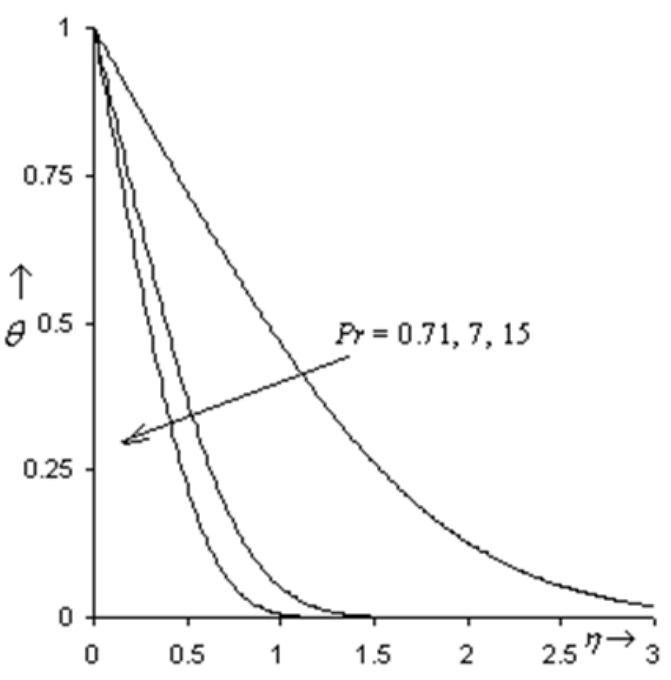

Fig. 12. Temperature distribution versus $\eta$ when $D a=0.2, G_{T}=1.0, G_{C}=0.5, K=1 \& S c=0.5$

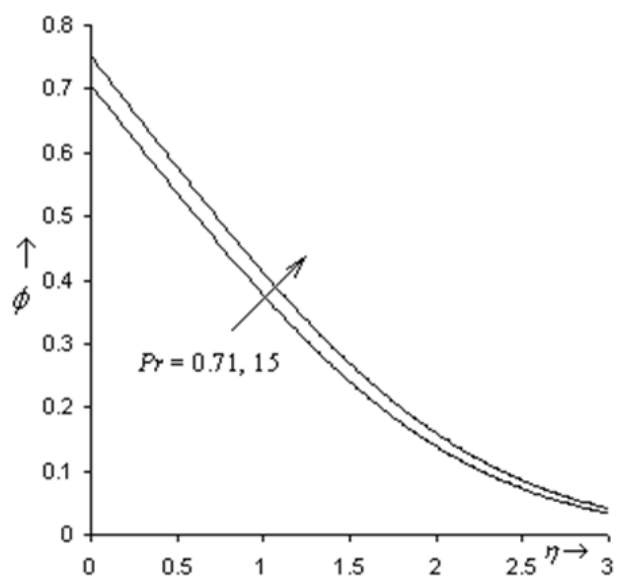

Fig. 13. Concentration distribution versus $\eta$ when $D a=0.2, G_{T}=1.0$ $G_{C}=0.5, K=1 \& S c=0.5$

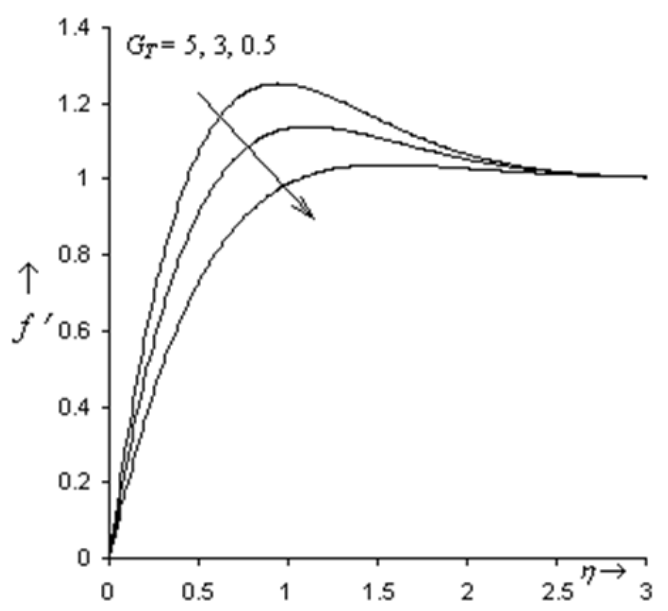

Fig. 14. Velocity distribution versus $\eta$ when $D a=0.2, G_{C}=0.5$, $K=1, S c=0.5 \& \operatorname{Pr}=1.0$

High fluid velocity for low $P r$ leads to decrease in specie concentration because of better diffusion, which is depicted in Fig. 13. The increase in $G_{T}$ means the 
increase of buoyancy force therefore the fluid velocity increases which in turn would convect heat and diffuse mass better in the fluid resulting decrease in fluid temperature and specie concentration and so the heat flux increase at the plate, while rate of mass flux decreases due to taken initial condition for specie at the plate. The similar explanation, as for increase in $G_{T}$ also holds for increase in $G_{C}$. These are presented in the Figs. 14-19 and Table 1.

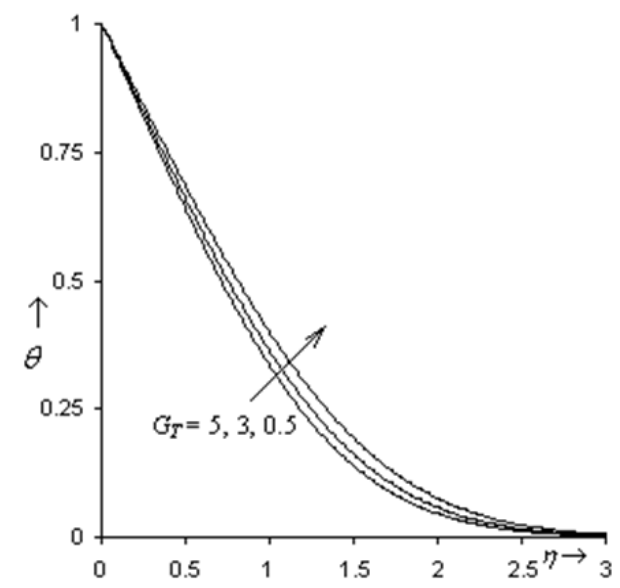

Fig. 15. Temperature distribution versus $\eta$ when $D a=0.2$, $G_{C}=0.5, K=1, S c=0.5 \& \operatorname{Pr}=1.0$

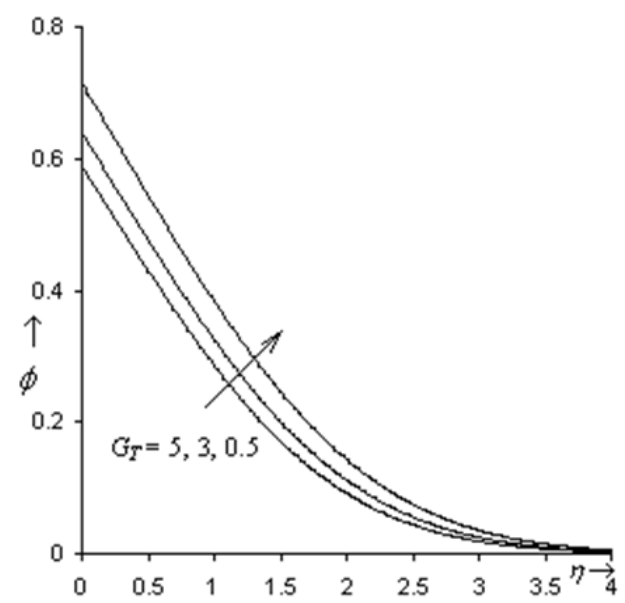

Fig. 16. Concentration distribution versus $\eta$ when $D a=0.2$, $G_{C}=0.5, K=1, S c=0.5 \& \operatorname{Pr}=1.0$

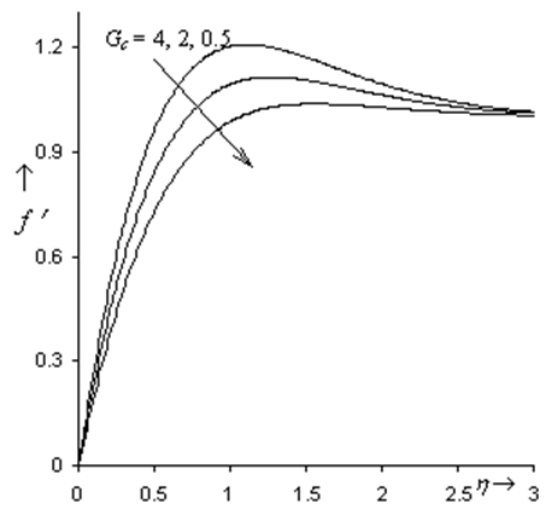

Fig. 17. Velocity distribution versus $\eta$ when $D a=0.2, G_{T}=1.0, K=1$, $\operatorname{Pr}=1.0 \& S c=0.5$

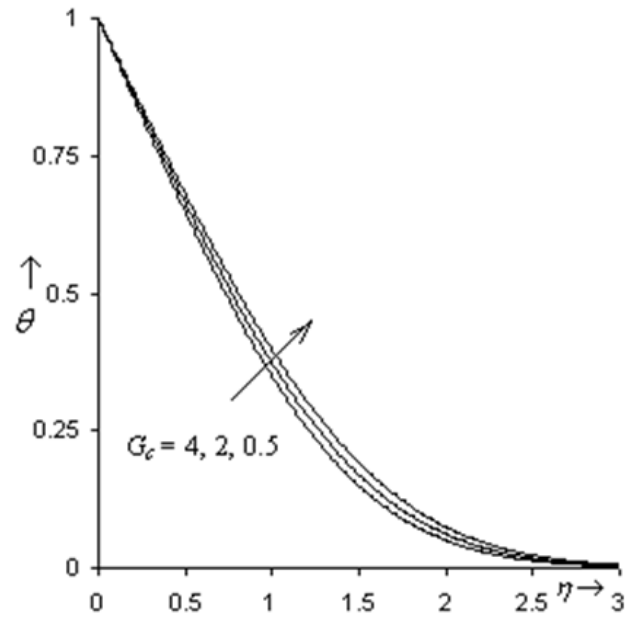

Fig. 18. Temperature distribution versus $\eta$ when $D a=0.2$, $G_{T}=1.0, K=1, \operatorname{Pr}=1.0 \& S c=0.5$

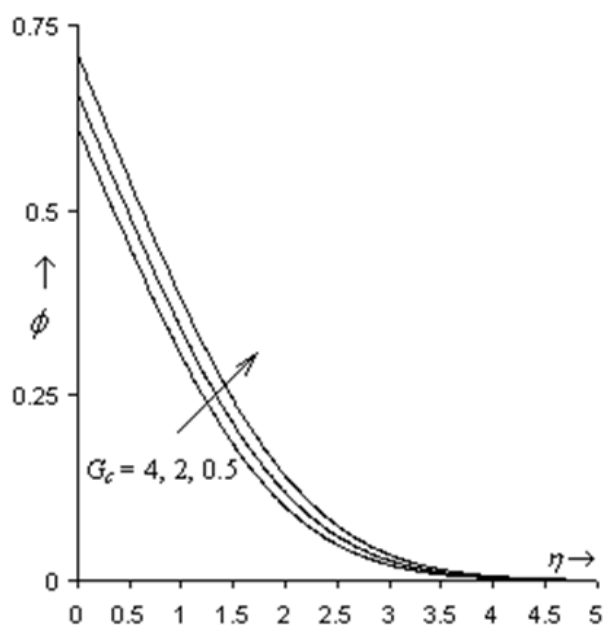

Fig. 19. Concentration distribution versus $\eta$ when $D a=0.2$, $G_{T}=1.0, K=1, P r=1.0 \& S c=0.5$

Table 2 shows that for given value of $D a$, system of differential Eqs. 8, 9 and 10 with boundary conditions Eq. (11) have two values of $f^{\prime \prime}(0), \theta^{\prime}(0), \phi^{\prime}(0), \phi(0)$ i.e. two solutions for one value of $D a$, which are called as first and second solution. It is seen in Table 2 that the first and second solution have positive value of $f^{\prime \prime}(0)$, which means that flowing fluid exerts drag on the static plate. The positive value of rate of heat transfer at plate i.e. $-\theta^{\prime}(0)>0$, depicts that heat transfer is from plate to fluid. Above two observations are in tune with the fluid flow and heat transfer phenomena. However, looking at the value of specie concentration at plate, $\phi(0)$, the second solution shows that $\phi(0)$ is negative which suggests that specie is consumed by the plate. This is practically impossible. The first solution has positive value for $\phi(0)$, which is acceptable. It is noteworthy in the case of first solution that specie concentration at plate rises aggressively with the increase in $D a$. Figs. 20, 21 and 22 show that both first and second solution are asymptotic in behavior and in accordance with boundary conditions and are thus mathematically correct. So, it can be suitably commented that although 
G. Singh and P. R. Sharma / JAFM, Vol. 7, No. 1, pp. 25-33, 2014.

the system has dual solution at higher $D a$, first solution is practically acceptable. Dual solutions in case of boundary layer flow have been reported earlier in literature by different authors. Chin et al. (2007), Ishak et al. (2008) have presented dual solution numerically using Keller Box Scheme for mixed convection flow. Here, it is important to point that Runge-Kutta fourth order method along with shooting technique is computationally able to capture the dual solution.

Table 2 Numerical value of $f^{\prime \prime}(0), \theta^{\prime}(0), \phi^{\prime}(0)$ and $\phi(0)$ for different value of parameter $D a$, showing existence of dual solution

\begin{tabular}{|c|c|c|c|c|c|}
\hline \multicolumn{2}{|c|}{} & \multicolumn{4}{|c|}{$G_{T}=1.0, G_{C}=0.5, K=1.0, P r=1.0, S c=0.5$} \\
\hline \multicolumn{2}{|c|}{$D a$} & $f^{\prime \prime}(0)$ & $-\theta^{\prime}(0)$ & $-\phi^{\prime}(0)$ & $\phi(0)$ \\
\hline \multirow{2}{*}{1.0} & $\mathrm{I}^{\text {st }} \mathrm{Sol}^{\mathrm{n}}$ & 10.8926 & 1.0169 & 60.1655 & 59.1655 \\
\cline { 2 - 6 } & $\mathrm{II}^{\text {nd }} \mathrm{Sol}^{\mathrm{n}}$ & 1.4907 & 0.5872 & -1.4229 & -2.4229 \\
\hline \multirow{2}{*}{1.1} & $\mathrm{I}^{\text {st }} \mathrm{Sol}^{\mathrm{n}}$ & 14.4656 & 1.1123 & 99.0541 & 89.0492 \\
\cline { 2 - 6 } & $\mathrm{II}^{\text {nd }} \mathrm{Sol}^{\mathrm{n}}$ & 1.5483 & 0.5922 & -1.2829 & -2.1662 \\
\hline \multirow{2}{*}{1.2} & $\mathrm{I}^{\text {st }} \mathrm{Sol}^{\mathrm{n}}$ & 18.7750 & 1.2093 & 155.1917 & 128.3264 \\
\cline { 2 - 6 } & $\mathrm{II}^{\text {nd }} \mathrm{Sol}^{\mathrm{n}}$ & 1.5885 & 0.5956 & -1.1825 & -1.9854 \\
\hline \multirow{2}{*}{1.3} & $\mathrm{I}^{\text {st }} \mathrm{Sol}^{\mathrm{n}}$ & 23.8797 & 1.3072 & 233.6301 & 178.7154 \\
\cline { 2 - 6 } & $\mathrm{II}^{\text {nd }} \mathrm{Sol}^{\mathrm{n}}$ & 1.6180 & 0.5980 & -1.1076 & -1.8520 \\
\hline \multirow{2}{*}{1.4} & $\mathrm{I}^{\text {st }} \mathrm{Sol}^{\mathrm{n}}$ & 29.8417 & 1.4057 & 340.3394 & 242.0996 \\
\cline { 2 - 6 } & $\mathrm{II}^{\text {nd }} \mathrm{Sol}^{\mathrm{n}}$ & 1.6405 & 0.5999 & -1.0498 & -1.7498 \\
\hline \multirow{2}{*}{1.5} & $\mathrm{I}^{\text {st }} \mathrm{Sol}^{\mathrm{n}}$ & 36.7243 & 1.5046 & 482.2821 & 320.5214 \\
\cline { 2 - 6 } & $\mathrm{II}^{\text {nd }} \mathrm{Sol}^{\mathrm{n}}$ & 1.6582 & 0.6014 & -1.0039 & -1.6692 \\
\hline
\end{tabular}

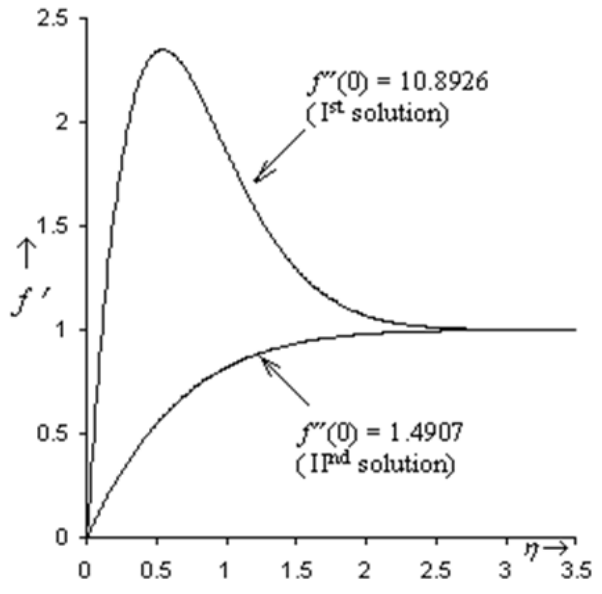

Fig. 20. Velocity distribution versus $\eta$ when $D a=1.0, G_{T}=1.0$, $G_{C}=0.5, K=1, \operatorname{Pr}=1.0 \& S c=0.5$

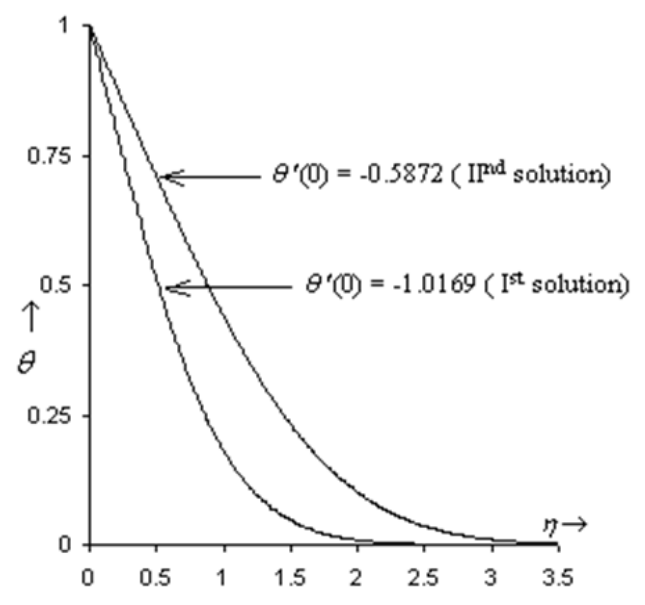

Fig. 21. Temperature distribution versus $\eta$ when $D a=1.0, G_{T}=1.0$, $G_{C}=0.5, K=1, P r=1.0 \& S c=0.5$ 


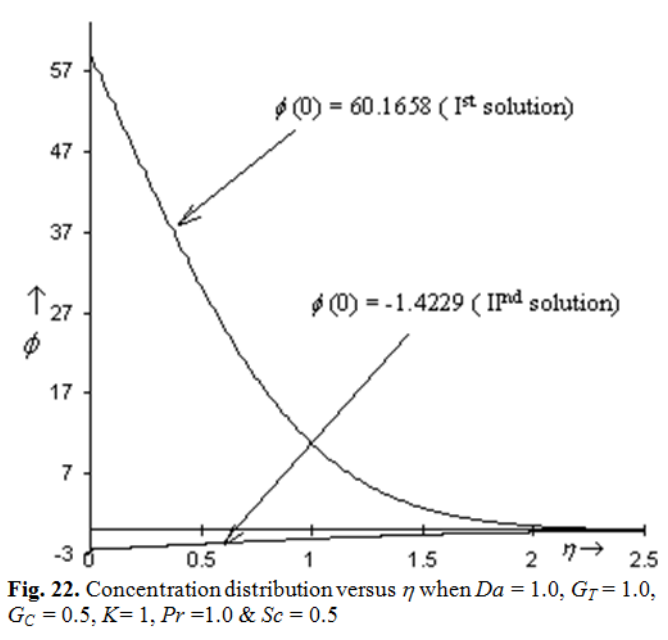

\section{Conclusion}

1. The skin friction increases coefficient with the increase in parameters $D a, K, G_{T}$ or $G_{C}$ and decreases with the increase in parameters $S c$ or $P r$.

2. The heat flux at plate increases with the increase in parameters $\mathrm{Da}, \mathrm{K}, \mathrm{Pr}, G_{T}$ or $G_{C}$ and decreases with the increase in parameter $S c$.

3. The specie mass flux and concentration at plate increase with the increase in parameters $\mathrm{Da}$ or $\mathrm{Pr}$ while decreases with the increase in parameters $\mathrm{Sc}$, $\mathrm{K}, G_{T}$ and $G_{C}$.

4. The fluid velocity increases with the increase in parameters, Da, K, $G_{T}$ or $G_{C}$ while decreases with the increase in parameters $S c$ or $P r$.

5. The fluid temperature increases with the increase in parameter $S c$, while decreases with increase in parameter $\mathrm{Da}, \mathrm{K}, \mathrm{Pr}, G_{T}$ or $G_{C}$.

6. The specie concentration in the fluid increases with increase in $D a$ or $P r$, while it decreases with the increase in parameters $S c, K, G_{T}$ or $G_{C}$.

The system of ordinary differential equations governing flow, heat and mass transfer has dual solution.

\section{REFERENCES}

Anson Chambre, P.L. and J.D. Young (1958). On the diffusion of a chemically reacting species in a laminar boundary layer, Physics of Fluids, 1, 4854.

Chamkha, A.J. and A.M. Aly (2011). Heat and mass transfer in stagnation point flow of a polar fluid towards a stretching surface in porous media in the presence of Soret, Dufour and chemical reaction effects, Chemical Engineering Communications, 198 (2), 214-234

Chamkha, A.J. and S.E. Ahmed (2009). Similarity solution for unsteady MHD flow near a stagnation point flow of a three dimensional porous body with heat and mass transfer, heat generation/absorption and chemical reaction, Journal of Applied Fluid Mechanics, 4(2), 87-94.

Chin, K.E., R. Nazar, N.M. Arifin and I. Pop (2007). Effect of variable viscosity on mixed convection boundary layer flow over a vertical surface embedded in porous medium, International Communnications in Heat and Mass Transfer, 34, 464-473.

Chin, T. (1975). Mass Transfer to continuous moving sheet electrode, Journal of Electrochemical Society, 122, 643-646.

Conte, S. D. and C. Boor (1981). Elementary Numerical Analysis. McGraw-Hill Book Co., New York.

Erickson, L.E., L.T. Fan and V.G. Fox (1966). Heat and mass transfer on a moving continuous flat plate with suction or injection, Industrial Engineering Chemical Fundamentals, 5, 19-25.

Hayat, T., S.A. Shehzad, A. Alsaedi and M.S. Alhothuali (2012). Mixed convection stagnation point flow of Casson fluid with convective boundary conditions, Chinese Physics Letters, 29(11), 114704 (1-4).

Ishak, A, R. Nazar, N.M. Arifin and I. Pop (2008). Dual solution in mixed convection flow near a stagnation point on a vertical porous plate, International Journal of Thermal Science, 47, 417422.

Liu, S., C.G. Fotache, B.H. Chao, D.J. Hautman and S.S. Ochs (2008). Boundary layer modeling of reactive flow over a porous surface with angled injection, Combustion and Flame, 154, 378-386.

Mahapatra, T.R. and S.K. Nandy (2013). Momentum and heat transfer in MHD axisymmetric stagnation point flow over a shrinking sheet, Journal of Applied Fluid Mechanics, 6(1),121-129.

Murray, P. and G. F. Carey (1998). Viscous flow and transport with moving free and reactive surfaces, International Journal for Numerical Methods in Engineering, 30, 1181-1194.

Sharma, P.R. and G. Singh (2010). Effect of variable thermal conductivity, viscous dissipation on steady MHD natural convection flow of low Prandtl fluid on an inclined porous plate with Ohmic heating, Meccanica, 45, 237-247.

Succi, S., G. Smith and E. Kaxiras (2002). Lattice Boltzmann Simulation of microflows over reactive surfaces, Journal of Statistical Physics, 107, 343366.

Wu, Q., S. Weinbaum and Y. Andreopoulos (2005). Stagnation point flow in a porous medium, Chemical Engineering Science, 60, 123-134.

Yih, K.A. (1998). The effect of uniform suction/blowing on heat transfer of magnetohydrodynamics Hiemenz flow though porous media, Acta Mechanica, 130, 147. 